\title{
A RELAÇÃo ENTRE PERCEPÇÃO DE BUROCRACIA E ESTRESSE NO SERVIÇO PÚBLICO FEDERAL
}

\author{
THE RELATION BETWEEN BUREAUCRACY PERCEPTION AND JOB STRESS IN BRAZILIAN \\ FEDERAL PUBLIC SERVICE
}

\section{GUSTAVO FREITAS PENA VIEIRA}

Mestrando em Gestão Pública - Universidade Federal do Espírito Santo (UFES)

E-mail: gfpvieira@gmail.com / Orcid: http://orcid.org/0000-0002-8637-5457

Endereço: R. Desembargador Augusto Botelho, 888, 304, Vila Velha - ES. CEP: 29.101-110

\section{IGOR REZENDE DE BARROS}

Mestrando em Gestão Pública - Universidade Federal do Espírito Santo (UFES)

E-mail: igorbarros@yahoo.com / Orcid: http://orcid.org/0000-0002-2153-4525

\section{DANILO MAGNO MARCHIORI \\ Professor do Mestrado Profissional em Gestão Pública da Universidade Federal do Espírito Santo (UFES) Doutorando em Gestão pela Universidade da Beira Interior (Portugal) E-mail: dmarchiori.ufes@gmail.com / Orcid: http://orcid.org/0000-0002-7386-9706}

\section{RESUMO}

O presente trabalho se dispõe a examinar dois fenômenos organizacionais largamente estudados, mas raramente relacionados, apesar de guardarem estreita proximidade. Há um vasto corpo de estudos acerca dos efeitos negativos da burocracia nas organizações. Em igual medida, inúmeras pesquisas buscam investigar as causas do estresse ocupacional. No entanto, carece a literatura nacional de investigação que questione a possível influência da percepção de burocracia no estresse experimentado pelos atores de uma organização. Visando preencher essa lacuna, foi realizada uma pesquisa que alcançou 293 servidores públicos de uma instituição federal de ensino superior. Os resultados evidenciam uma correlação bem definida, ainda que pequena, entre a percepção de burocracia e o estresse experimentado, demonstrando a relevância de se levar em conta a percepção de burocracia como um possível estressor no estudo do estresse ocupacional.

Palavras-chave: Burocracia. Estresse. Gestão Pública. Correlação. Regressão.

\begin{abstract}
In this article, we analyze two widely studied, but rarely related, organizational phenomena: bureaucracy and stress. There is vast literature about the negative effects of bureaucracy in organizations. In equal measure, many studies try to investigate the causes of job stress. However, there is a lack of research, at least on Brazilian context, about the possible relation between the perception of bureaucracy and the feeling of stress on the organizational field. Trying to fill this gap, we examined the data from a survey based on a sample of 293 public servants of a federal university. The empirical results show that bureaucracy might be a relevant factor in the study of job stress.
\end{abstract}

Keywords: Bureaucracy. Stress. Public Service. Correlation. Regression. 


\section{INTRODUÇÃO}

No estudo da ciência administrativa, burocracia é um dos temas de abordagem praticamente obrigatória. O fenômeno tem como seu mais reconhecido teórico Max Weber (1864-1920), que abordou o instituto de uma perspectiva científica e imparcial (TENÓRIO, 1981).

O "tipo ideal" da burocracia weberiana, baseado na autoridade legal ou racional, se caracteriza pela prevalência de normas escritas, documentação da atividade, divisão sistematizada do trabalho, regras e normas técnicas rígidas e uma hierarquia e controle bem definidos (WEBER, 1999).

A idealização da burocracia surgiu, então, como forma de se alcançar eficiência técnica e de evitar abusos discricionários (sem observância das normas) por parte dos agentes organizacionais. Isso não evitou, entretanto, que o tempo degenerasse as técnicas burocráticas em rotinas disfuncionais (TENÓRIO, 1981).

Como será demonstrado no referencial teórico a seguir, diversos estudos vêm evidenciando que as práticas burocráticas atuais, muitas vezes distantes do tipo ideal proposto por Weber, vem acarretando efeitos nocivos e sentimentos negativos no contexto organizacional.

De conceituação mais recente, o estresse é um fenômeno organizacional que vem sendo largamente estudado nos dias de hoje, em especial o estresse relacionado ao trabalho, também chamado estresse ocupacional.

Tema em voga e de grande relevância para o campo da administração, o estresse ocupacional é conceituado como percepção ou sentimento individual de disfunção fisiológica ou psicológica decorrente de condições ou acontecimentos relacionados ao trabalho (PARKER; DECOTIIS, 1983).

A ser demonstrado adiante, o estresse relacionado ao trabalho, enquanto sentimento negativo, pode se originar de diversas causas (estressores). A percepção de burocracia, por sua vez, tem sido relacionada a uma vasta gama de efeitos desfavoráveis. Assim, salta aos olhos a possibilidade de que os dois fenômenos, ambos presentes no contexto organizacional, possam estar relacionados.

Não obstante a aparente clareza da constatação, existe uma lacuna, ao menos na literatura nacional, quanto a essa investigação. Diversos estudos se ocuparam dos efeitos da burocracia. (LYRIO; DELLAGNELO; LUNKES, 2017; PIRES, 2009; SANTOS; MARQUES 2015; SARAIVA, 2002; SARAIVA; CAPELÃO, 2000). Outros tantos, das causas e dos impactos do estresse nas organizações (ALMEIDA et al., 2018; BALASSIANO; TAVARES; PIMENTA, 2011; BARCAUI; LIMONGI-FRANÇA, 2014; MAFFIA; PEREIRA, 2014; PAIVA; COUTO, 2008; TAMAYO, 2001). Mas faltam pesquisas que apurem, especificamente, se existe relação, ou não, entre a percepção da burocracia e o estresse experimentado pelos atores organizacionais.

Impulsionado por essa inquietação, pretende o presente estudo analisar a relação entre percepção de burocracia e estresse ocupacional. Para tanto, foi testada a hipótese com uma amostra de 293 servidores públicos de uma instituição federal de ensino superior.

$O$ trabalho é estruturado em seis seções. A primeira seção introduz o assunto e expõe a inquietação que impulsionou a presente pesquisa. Na segunda, apresenta-se o referencial teórico acerca da burocracia, do estresse ocupacional, e desses fenômenos no âmbito das organizações públicas. Nas três seções seguintes, demonstra-se o método pelo qual a pesquisa foi realizada, segue-se com a apresentação dos resultados e, após, com a discussão dos mesmos. Por fim, na sexta e última seção, são apresentadas as considerações finais e as possíveis contribuições do trabalho realizado para o campo da ciência administrativa. 


\section{REFERENCIAL TEÓRICO}

A fim de inserir o presente estudo no contexto da produção científica e contribuir para a compreensão das análises realizadas e resultados encontrados, realizou-se uma revisão da literatura sobre os temas abordados no estudo: burocracia e estresse ocupacional.

\subsection{BUROCRACIA}

O termo burocracia carrega um teor pejorativo desde a sua gênese, quando foi empregado para denominar o corpo de funcionários do Estado absolutista francês sob a dependência do soberano. Ainda hoje, não é rara a associação do vocábulo com excesso de formalismo, abundância de papéis, rotinas intrincadas e sem finalidade e rigorosidade das normas (ARAGÃO, 1997; PIZZA JR., 1984).

Inicialmente, já é possível constatar que o sentimento antiburocrático difundido na população pode deteriorar a moral dos servidores públicos e tornar-se fonte de descrença e insatisfação (KIM; FERNANDEZ, 2016).

Igualmente comum é a associação entre burocracia e ineficiência (ARAGÃO, 1997). A demanda das organizações por aderência às normas leva a um deslocamento de objetivos: as regras se tornam um fim em si mesmas, e a adesão a procedimentos formalizados dificulta a adaptação das regras às circunstâncias especiais. Com isso, as regras pensadas para produzir eficiência acabam, do contrário, produzindo ineficiência. (BOZEMAN; SCOTT, 1996).

Adaptar-se a um sistema burocrático complexo e impessoal é um pesado encargo, apto a gerar sentimentos de ansiedade e alienação. Processos excessivamente impessoais e rotinizados, que desprezam os aspectos pessoais, são capazes de produzir sentimentos generalizados de impotência frente às grandes instituições, bem como frustrar a criatividade e o desenvolvimento pessoal (SMITH, 1971). "O emprego rigoroso de prescrições formais e a rigidez da hierarquia deixam marcas na alma dos que são responsáveis pela sua legitimação" (PIZZA JR., 1984, p. 12).

A burocracia excessiva tem sido reconhecida em diversos estudos como fonte de sentimentos negativos. DeHart-Davis apresentou evidência de que gestores públicos em organizações mais centralizadas tendem a experimentar maior grau de alienação (estado psicológico de desconexão com o trabalho) do que aqueles em organizações menos centralizadas (DEHART-DAVIS, 2005). O excesso de formalização burocrática tem relação com sentimentos negativos como alienação e impotência (PANDEY; KINGSLEY, 2000).

Zeffane, na mesma linha, assevera que há um grande corpo de pesquisa evidenciando que os empregados se sentem menos alienados quando são autorizados a participar de decisões sobre o seu trabalho, sendo colaboradores ao invés de ferramentas de gestão. (ZEFFANE, 1993). É o contrário do que propõe a prática burocrática, que privilegia a centralização em detrimento da participação.

Há evidência de que maiores níveis de burocratização estão associados com menor satisfação procedimental percebida (satisfação com os processos). Vários estudos têm demonstrado que a satisfação tem implicações importantes tanto para a performance organizacional quanto para os níveis individuais de motivação e confiança (KAUFMANN; TUMMERS, 2016).

A estagnação, ou falta de inovação, é outro ponto percebido. Moon e Bretschneiber asseveram que níveis excessivos de burocratização tendem a atrasar e ou até impedir a inovação, especialmente quando a organização se encontra sobrecarregada por procedimentos administrativos (MOON; BRETSCHNEIBER, 2002). Crozier chega a definir como 
organização burocrática aquela que não consegue se corrigir em função de seus erros, tendendo, do contrário, à perpetuação das práticas, ainda que incorretas. (CROZIER, 1981).

Outro aspecto da burocracia, em especial no serviço público, é a possibilidade que tem o agente público de ser legalmente controlado, monitorado e/ou punido por um ator ou órgão de controle, o que, em inglês, se denomina accountability (OLIVIERI, 2011). Bozeman e Scott demonstram que a sujeição dos agentes de organizações públicas a maiores níveis de responsabilização legal (accountability) os submete a maiores restrições operacionais, regras mais pesarosas e percepção de formalização excessiva (BOZEMAN; SCOTT, 1996).

O espectro mais patológico da burocracia foi batizado, na língua inglesa, de red tape. Bozeman conceitua red tape como o conjunto de regras, regulamentos e procedimentos cuja obediência é obrigatória dentro da organização, mas que não tem nenhuma eficiência quanto aos propósitos que deveriam atender (BOZEMAN; SCOTT, 1996). Segundo Pandey e Scott, red tape é a percepção dos gestores de que a formalização (na forma de uma pesada carga de normas e regulamentos) é prejudicial para os propósitos da organização (PANDEY; SCOTT, 2002).

Inúmeros estudos apresentam evidência de que a percepção desses aspectos mais prejudiciais da burocracia (red tape) está relacionada a atitudes negativas, como falta de motivação (BALDWIN, 1990) e crescente insatisfação (MOYNIHAN; PANDEY, 2007). Há indícios, também, de que diferentes tipos de red tape afetam negativamente a performance dos indivíduos nas organizações (BREWER; WALKER, 2010; PANDEY, MOYNIHAN, 2006).

Assim, demonstrando a literatura científica que a percepção da burocracia tem associação com sentimentos negativos, passa-se à análise de um sentimento prejudicial de grande relevância no meio organizacional: o estresse ocupacional.

\subsection{ESTRESSE OCUPACIONAL}

O estresse ocupacional descreve o sentimento de uma pessoa que é obrigada a desviar do seu estado de funcionamento normal ou desejável como resultado de oportunidades, restrições, ou demandas relacionadas ao trabalho. A conceituação do estresse ocupacional como sendo desconfortável e indesejável ajuda a distingui-lo do sentimento positivo que pode resultar do desafio de se atingir um objetivo difícil, mas alcançável. (PARKER; DECOTIIS, 1983).

Um problema que interfere na construção da literatura acerca do fenômeno do estresse é a multicolinearidade inerente ao grande número de variáveis tipicamente associadas com o estresse ocupacional (PARKER; DECOTIIS, 1983). De fato, o rol de fatores aptos a causar estresse não é consensual, nem tampouco exaustivo.

Cooper e Marshal definiram cinco fontes de estresse relacionadas ao trabalho: (1) fatores intrínsecos ao trabalho, como sobrecarga de informação ou pressão dos prazos, (2) papel na organização, (3) desenvolvimento na carreira, (4) relacionamento no trabalho, (5) estrutura e clima organizacional, aqui incluindo baixo envolvimento na tomada de decisões (COOPER; MARSHALL, 1976).

Parker e DeCotiis, por sua vez, entenderam que o estresse ocupacional pode decorrer de 6 categorias de estressores: (1) características e condições do trabalho em si, (2) condições associadas com a estrutura, clima e fluxo de informação na organização, (3) papel desempenhado na organização, (4) relacionamentos no trabalho, (5) percepção do desenvolvimento na carreira, (6) compromissos e responsabilidades externas. (PARKER; DECOTIIS, 1983).

Em verdade, existe a possibilidade de que o fenômeno do estresse seja muito complexo para admitir uma teoria que especifique somente um pequeno conjunto de 
variáveis que cause o conteúdo, o processo e as consequências do estresse (PARKER; DECOTIIS, 1983). Vislumbra-se, assim, a possibilidade de que a percepção de burocracia possa também ser uma fonte de estresse ocupacional, podendo facilmente estar inserida em uma das categorias elencadas nos parágrafos anteriores.

Há um vasto corpo de estudos evidenciando que o estresse ocupacional afeta negativamente a performance dos indivíduos no trabalho (BAKKER; VAN EMMERIK; VAN RIET, 2008; HAMANN; FOSTER, 2013), o comprometimento organizacional, a satisfação no trabalho (ALMEIDA et al., 2018; PARKER; DECOTIIS, 1983) e é um fator determinante na intenção de deixar o serviço público (KIM, 2005).

Dada a relevância de suas consequências, torna-se importante, para o gerenciamento do estresse ocupacional, a correta identificação dos estressores organizacionais (BALASSIANO; TAVARES; PIMENTA, 2011; MAKHIJA 2016). O sucesso de qualquer esforço para minimizar o estresse e maximizar a satisfação no trabalho depende deste diagnóstico (BALASSIANO; TAVARES; PIMENTA, 2011).

Apesar da lacuna de pesquisa que avalie especificamente a percepção de burocracia como estressor determinante para o estresse ocupacional, alguns estudos apontam, indiretamente, nesse sentido.

Sert e colaboradores, assim como Santos e colaboradores, apresentaram evidências de que quando empregados são forçados a obedecer a regras, políticas e procedimentos que eles acreditam serem injustas, surgem sérios problemas no local de trabalho associados com estresse ocupacional (SANTOS; OLIVEIRA; KONOPKA, 2016; SERT et al., 2014). A percepção de disfunções da burocracia pode ser interpretada pelos funcionários como regras injustas.

Há evidência de que o estresse pode ser determinado por variáveis como autonomia, abertura da comunicação e formalização, (PARKER; DECOTIIS, 1983) fatores determinantes na caracterização de uma organização burocrática.

Fatores intrínsecos ao trabalho e relacionados ao papel do indivíduo na organização também foram evidenciados como estressores (BALASSIANO; TAVARES; PIMENTA, 2011), e a burocracia tende a se relacionar a esses dois aspetos.

Karasek sugere que trabalhadores em empregos com alta demanda de trabalho são os mais estressados e menos satisfeitos (KARASEK, 1979). No entanto, há mecanismos que podem mitigar o efeito da alta demanda de trabalho, como participação na tomada de decisão e autonomia (HAMANN; FOSTER, 2013). Esses mecanismos de mitigação são normalmente ausentes em organizações com altos níveis de burocracia, que privilegiam a centralização das decisões no lugar da participação e o controle rígido no lugar da autonomia.

Assim, a produção acadêmica deixa clara a abertura na definição dos estressores determinantes para o estresse ocupacional. E havendo indícios, ao menos indiretos, de que a percepção da burocracia pode se caracterizar como um de tantos estressores possíveis, tornase imperativa a investigação nesse sentido, a fim de suprir a lacuna existente.

\subsection{ESTRESSE E BUROCRACIA NAS ORGANIZAÇÕES PÚBLICAS}

Optou-se por iniciar a investigação da relação entre burocracia e estresse ocupacional no contexto de uma instituição pública federal. Há indícios de que a percepção de burocratização excessiva tende a ser mais alta nas organizações públicas do que nas privadas, especialmente por conta do elevado grau de controle externo e responsabilização legal (accountability) (BREWER; WALKER, 2010). Pandey e Kingsley apresentam evidências de que gestores públicos tendem a perceber maiores níveis de formalização burocrática do que seus correspondentes da iniciativa privada (PANDEY; KINGSLEY, 2000). Saraiva demonstra que a 
burocracia é o fator de racionalidade predominante na esfera pública (SARAIVA; CAPELÃO, 2000).

Há, também, indicações empíricas da presença de estresse entre empregados do serviço público (KIM, 2005). Em certas áreas, como na área da enfermagem, é possível, inclusive, encontrar evidências de maior nível de estresse entre os trabalhadores do setor público em relação aos seus correspondentes do setor privado (HAMANN; FOSTER, 2013).

Aliado ao fato de que, no campo da administração pública, ainda se percebe uma carência de pesquisas sobre o estresse ocupacional (BALASSIANO; TAVARES; PIMENTA, 2011), optou-se por esse nicho de pesquisa, com a propositura da seguinte hipótese:

$\mathbf{H}_{1}$ : A percepção de burocracia tem relação direta com 0 estresse experimentado pelos servidores públicos federais.

\section{MÉTODO}

A amostra analisada constitui-se de 293 servidores públicos de uma instituição federal de ensino superior ( $N=293$ ). Esse número representa uma amostra diversificada de respondentes dentre todos os servidores ativos de uma universidade federal, que foram consultados através de um formulário online encaminhado via e-mail institucional.

A universidade consta com 3.811 servidores ativos permanentes, distribuídos entre seus quatro campi universitários em três municípios do estado do Espírito Santo: Vitória, Alegre e São Mateus. A taxa de respondentes correspondeu a aproximadamente $8 \%$ dos servidores consultados. A amostra foi demograficamente diversificada, tanto por sexo, quanto por idade, nível educacional e tempo de atuação no órgão, conforme apresentado na Tabela 1. As variáveis objeto da pesquisa foram analisadas através de uma escala de 5 pontos do tipo Likert, ranqueando desde 1 = discordo totalmente, até 5 = concordo totalmente.

Tabela 1: Informações demográficas

\begin{tabular}{|c|c|c|c|}
\hline & & Frequência & Porcentagem \\
\hline \multirow{3}{*}{ Sexo } & Homem & 142 & 48,5 \\
\hline & Mulher & 151 & 51,5 \\
\hline & Total & 293 & 100 \\
\hline \multirow{8}{*}{ Idade } & 18-24 anos & 3 & 1 \\
\hline & $25-30$ anos & 55 & 18,8 \\
\hline & $31-35$ anos & 65 & 22,2 \\
\hline & $36-40$ anos & 68 & 23,2 \\
\hline & 41-45 anos & 40 & 13,7 \\
\hline & 46-50 anos & 22 & 7,5 \\
\hline & 50 ou mais & 40 & 13,7 \\
\hline & Total & 293 & 100 \\
\hline \multirow{6}{*}{ Escolaridade } & Doutorado & 102 & 34,8 \\
\hline & Ensino Médio & 2 & 0,7 \\
\hline & Ensino Superior & 45 & 15,4 \\
\hline & Especialização & 72 & 24,6 \\
\hline & Mestrado & 72 & 24,6 \\
\hline & Total & 293 & 100 \\
\hline \multirow{5}{*}{ Tempo de Trabalho } & 11-15 anos & 23 & 7,8 \\
\hline & 6-10 anos & 98 & 33,4 \\
\hline & Mais que 15 anos & 37 & 12,6 \\
\hline & Menos que 5 anos & 135 & 46,1 \\
\hline & Total & 293 & 100 \\
\hline
\end{tabular}

Fonte: dados da pesquisa. 
A variável independente percepção de burocracia foi mensurada através da escala proposta por Wright (2004), composta por três itens:

No meu trabalho, mesmo questões pequenas devem ser encaminhadas para alguém superior para uma aprovação final.

Eu sempre devo verificar com meu chefe antes de tomar decisões importantes. Esta organização parece muito mais preocupada que eu siga os procedimentos de que faça um bom trabalho.

A confiabilidade estimada pelo alfa de Cronbach foi de ,707. A variável dependente estresse percebido foi mensurada através da escala proposta por Lambert et al. (2006), composta por cinco itens:

Meu trabalho me deixa muito frustrado ou irritado, a maior parte do tempo. Geralmente estou sob muita pressão quando estou no trabalho.

Muitas vezes me sinto tenso ou irritado quando estou no trabalho.

Eu costumo ficar tranquilo e à vontade quando estou trabalhando (reverso). Há muitos aspectos do meu trabalho que me deixam chateado.

A confiabilidade estimada pelo alfa de Cronbach foi de ,858. O questionário elaborado foi submetido a um pré-teste com 8 respondentes, que aprovaram a redação do mesmo. 0 questionário foi submetido por meio de formulário eletrônico (Google Forms) e encaminhado ao e-mail funcional de todos os servidores ativos permanentes da universidade federal. As respostas, dadas voluntariamente, foram coletadas em um período de 5 (cinco) dias úteis, de segunda a sexta-feira.

De posse dos dados oriundos dos questionários respondidos, utilizou-se o software SPSS 22 para testar a hipótese proposta. Inicialmente, foi realizada uma análise de correlação entre as variáveis, tendo em vista o potencial da técnica para mensurar a força da associação linear de duas variáveis (HAIR, 2005).

Em seguida, foi aplicada uma análise de regressão linear bivariada (ou simples) para avaliar a dependência da variável "estresse" em relação à variável independente "burocracia". Justifica-se a adoção da técnica da regressão, tendo em vista que a análise de correlação indica a potencial existência de uma relação entre duas variáveis, incluindo a intensidade dessa ligação, mas não é capaz de ser utilizada para realizar previsões. Assim, segundo Hair (2005), a regressão linear bivariada complementa os resultados obtidos por meio de uma análise de correlação, ao acrescentar informações sobre a relação entre uma variável independente (ou indicador) e uma variável dependente (ou critério). Dessa forma, é possível prever o comportamento da variável dependente a partir da variável independente.

A fim de validar os resultados do modelo de regressão, foram realizados os diagnósticos de homogeneidade dos resíduos, e distribuição normal dos erros e colinearidade, sem identificação de problemas.

\section{RESULTADOS}

A medição da escala "percepção de burocracia" retornou uma percepção média de burocracia da ordem de 3,2696, em uma escala de 1 a 5, conforme Tabela 2.

Tabela 2: Estatísticas descritivas - percepção de burocracia

\begin{tabular}{ccccc}
\hline & Bur1 & Bur2 & Bur3 & Burocracia (média) \\
\hline Média & 2,945 & 3,464 & 3,399 & 3,270 \\
Desvio Padrão & 1,386 & 1,336 & 1,332 & 1,073 \\
\hline
\end{tabular}

Fonte: dados da pesquisa. 
A medição da escala "estresse percebido", por sua vez, retornou uma percepção média de estresse por parte dos servidores equivalente a 2,911, como demonstra a Tabela 3.

Tabela 3: Estatísticas descritivas - estresse percebido

\begin{tabular}{ccccccc}
\hline & Est1 & Est2 & Est3 & Est4 & Est5 & Estresse (médio) \\
\hline Média & 2,652 & 2,696 & 2,816 & 2,8532 & 3,539 & 2,911 \\
Desvio Padrão & 1,183 & 1,191 & 1,250 & 1,157 & 1,251 & 0,964 \\
\hline
\end{tabular}

Fonte: dados da pesquisa.

A análise de correlação entre as variáveis "percepção de burocracia" e "estresse percebido" resultou em um coeficiente de Pearson $(r)$ da ordem de ,290, indicando uma correlação positiva pequena, mas definida (HAIR, 2005), com um sig $(p)<0,01$ e um nível de confiança de $99 \%$, conforme tabela 4.

Tabela 4: Correlação Estresse-Burocracia

\begin{tabular}{ccc}
\hline & & Burocracia \\
\hline \multirow{2}{*}{ Estresse } & Correlação de Pearson &, $290^{* *}$ \\
& Sig. (2 extremidades) & 0 \\
$\mathrm{~N}$ & 293 \\
\hline
\end{tabular}

**. A correlação é significativa no nível 0,01 ( 2 extremidades).

Fonte: dados da pesquisa.

A análise regressiva linear bivariada demonstrou um coeficiente de determinação $\left(R^{2}\right.$ ajustado) de ,081, indicando que o modelo explica $8,1 \%$ da variação da variável dependente, com um sig $(p)<0,01$ e um nível de confiança de $99 \%$, como demonstram as tabelas 5 e 6 .

Tabela 5: Resumo do modelo

\begin{tabular}{ccccc}
\hline & & \multicolumn{3}{c}{ Modelo $^{\mathrm{b}}$} \\
\hline Modelo & $\mathrm{R}$ & $\begin{array}{c}\mathrm{R} \\
\text { quadrado }\end{array}$ & $\begin{array}{c}\text { R quadrado } \\
\text { ajustado }\end{array}$ & Erro padrão da estimativa \\
\hline 1 &, $290^{\mathrm{a}}$ & 0,084 & 0,081 & 0,924 \\
\hline
\end{tabular}

a. Preditores: (Constante), Burocracia

b. Variável Dependente: Estresse

Fonte: dados da pesquisa.

Tabela 6: Coeficientes do modelo

\begin{tabular}{|c|c|c|c|c|c|c|c|c|}
\hline \multicolumn{9}{|c|}{ Coeficientes $^{a}$} \\
\hline \multirow{2}{*}{\multicolumn{2}{|c|}{ Modelo }} & \multicolumn{2}{|c|}{$\begin{array}{l}\text { Coeficientes } \\
\text { não } \\
\text { padronizados }\end{array}$} & \multirow{2}{*}{$\begin{array}{c}\text { Coeficientes } \\
\text { padronizados } \\
\text { Beta }\end{array}$} & \multirow[t]{2}{*}{$\mathrm{t}$} & \multirow[t]{2}{*}{ Sig. } & \multicolumn{2}{|c|}{$\begin{array}{l}\text { Estatísticas de } \\
\text { colinearidade }\end{array}$} \\
\hline & & B & $\begin{array}{l}\text { Erro } \\
\text { Padrão }\end{array}$ & & & & Tolerância & VIF \\
\hline \multirow{2}{*}{1} & (Constante) & 2,058 & 0,173 & & 11,872 & ,000 & & \\
\hline & Burocracia & 0,261 & 0,050 & 0,290 & 5,176 & ,000 & 1,000 & 1,000 \\
\hline
\end{tabular}

a. Variável Dependente: Estresse

Fonte: dados da pesquisa. 


\section{DISCUSSÃO}

Os resultados apresentados oferecem um suporte confiável para a hipótese proposta. Há evidência de uma correlação bem definida, ainda que pequena, entre a percepção de burocracia e o estresse ocupacional percebido, ao menos no contexto de uma instituição federal de ensino superior.

O modelo de que a percepção de burocracia é capaz de explicar o estresse ocupacional experimentado foi capaz de prever $8,1 \%$ da variação do estresse em relação à burocracia percebida, com um grau de confiança de $99 \%$ (sig. < 0,01). Nunca foi a pretensão do estudo propor um modelo de previsão para o estresse ocupacional. Fenômenos tão complexos não podem ser explicados por um conjunto tão restrito de apenas duas variáveis. Mas a constatação de que uma única variável, a burocracia, consegue explicar a variação de $8,1 \%$ do estresse percebido corrobora a hipótese de que existe uma relevante relação entre os dois fenômenos.

Detectada a relação, fornece-se aos gestores públicos o vislumbre de um fator a ser gerenciado (o nível de burocracia) quando do tratamento do estresse ocupacional na organização. Tendo se apresentado como potencial estressor, a implantação de algumas práticas alternativas à rigidez burocrática, como descentralizar decisões, dar maior oportunidade de participação e autonomia aos funcionários e servidores, flexibilizar normas procedimentais desnecessariamente enrijecidas, ampliar os canais de diálogo e incentivar a inovação, podem ter um impacto positivo na percepção do estresse, e, assim, mitigar os seus efeitos nocivos na instituição.

Certamente o estudo apresenta limitações. Em primeiro lugar, apesar de seu tamanho, a amostra pesquisada se restringe a uma única organização pública federal. Características peculiares da instituição podem ter influência nos resultados, especialmente quando as variáveis dependem da percepção dos integrantes da organização.

A percepção em si é uma segunda limitação. No caso da burocracia, escalas objetivas, e não baseadas na percepção do indivíduo, podem oferecer medidas mais exatas da quantidade de burocracia em uma organização. Apesar de, para o presente trabalho, a percepção ser importante, dada a tentativa de se relacionar a burocracia a um sentimento (estresse), e, como propõe a filosofia de Schopenhauer, o indivíduo só sente aquilo que percebe (WARBURTON, 2016).

Quanto ao estresse ocupacional, certamente é problemático ter que medir tanto o estresse quanto os estressores pela lente da percepção do indivíduo. A influência de características subjetivas na medição é inevitável. No entanto, não parece haver alternativa que não fuja da precisão do conceito de estresse. (PARKER; DECOTIIS, 1983).

Ademais, merece atenção a circunscrição da presente pesquisa apenas ao âmbito público. De fato, a replicação de pesquisas como essa em organizações do setor privado é importante para fins de comparação, com o intuito de controlar se características peculiares das organizações públicas e privadas são capazes influir nos fenômenos da burocracia e do estresse ocupacional.

\section{CONSIDERAÇÕES FINAIS}

O presente trabalho traz evidências de que a percepção de burocracia é capaz de influenciar no estresse experimentado pelos agentes de uma organização. Preenchendo uma lacuna investigativa, o resultado apresentado vem enriquecer a literatura no campo da ciência administrativa, tanto a que trata dos efeitos da burocracia, quando a que se ocupa das causas do estresse ocupacional. 
Os resultados do presente estudo indicam que, no campo prático, os gestores empenhados em melhorar o ambiente organizacional não devem deixar de incluir ações concretas com o objetivo de reduzir a burocracia dos processos de trabalho. Além disso, recomenda-se que os instrumentos utilizados pelas organizações para medir o estresse ocupacional recebam o acréscimo da dimensão burocracia. No campo acadêmico, as novas pesquisas envolvendo os antecedentes do estresse ocupacional poderão acrescentar a dimensão burocracia, a fim de elevar o poder explicativo dos modelos, bem como confrontar os resultados com os encontrados na presente pesquisa.

O caráter novel da pesquisa clama aos trabalhos futuros que prossigam na investigação da percepção de burocracia como um possível estressor, a ser levado em conta pelos gestores organizacionais quando do gerenciamento do fenômeno do estresse ocupacional. Caso a relação seja corroborada, uma importante adição se apresenta, devendo a burocracia ser inserida no rol de desafios a serem superados no gerenciamento do bem-estar organizacional.

No entanto, caso pesquisas futuras refutem a hipótese aqui levantada, igualmente caminha a ciência da administração. Surgindo demonstrações de que a burocracia não é causadora de estresse, pode-se estar diante de valiosos argumentos em defesa do "lado bom" da prática burocrática. É possível que aspectos da burocracia, como a redução de incertezas (ZEFFANE, 1993), a clareza de responsabilização, a previsibilidade, os freios à discricionariedade (BOZEMAN; SCOTT, 1996) e o fortalecimento das atividades meio das organizações (ANDREWS; BOYNE; MOSTAFA, 2017)) tenham um efeito positivo sobre o estresse ocupacional.

Qualquer que seja o caso, a investigação da hipótese levantada é enriquecedora para a ciência da administração, oferecendo subsídios adicionais para a compreensão de fenômenos organizacionais de grande importância: burocracia e estresse ocupacional.

\section{REFERÊNCIAS}

ALMEIDA, D. M.; LOPES, L. F. D.; COSTA, V. M. F.; SANTOS, R. C. T. Policiais militares do Estado do RS: relação entre satisfação no trabalho e estresse ocupacional. Administração Pública e Gestão Social, Viçosa - MG, p.55-65, 1 jan. 2018. Administração Pública e Gestão Social. DOI: http://dx.doi.org/10.21118/apgs.v10i1.1366.

ANDREWS, R.; BOYNE, G.; MOSTAFA, A. M. S. When bureaucracy matters for organizational performance: exploring the benefits of administrative intensity in big and complex organizations. Public Administration, [s.I.], v. 95, n. 1, p.115-139, fev. 2017. DOI: http://dx.doi.org/10.1111/padm.12305.

ARAGÃO, C. V. Burocracia, eficiência e modelos de gestão pública: um ensaio. Revista de Serviço Público, Brasília, ano 48, n. 3, p. 104-132, set-dez, 1997. DOI: https://doi.org/10.21874/rsp.v48i3.391.

BAKKER, A. B.; VAN EMMERIK, H.; VAN RIET, P. How job demands, resources, and burnout predict objective performance: A constructive replication. Anxiety, Stress \& Coping, [s.I.], v. 21, n. 3, p.309-324, jul. 2008. DOI: http://dx.doi.org/10.1080/10615800801958637.

BALDWIN, P. The politics of social solidarity: class bases of the European welfare state, 1875-1975. Cambridge, UK: Cambridge University Press, 1990. 
BARCAUI, A.; LIMONGI-FRANÇA, A. C. Estresse, enfrentamento e qualidade de vida: um estudo sobre gerentes brasileiros. Revista de Administração Contemporânea, Curitiba, v. 18, n. 5, p.670-694, set. 2014. DOI: http://dx.doi.org/10.1590/1982-7849rac20141865.

BALASSIANO, M.; TAVARES, E.; PIMENTA, R. C. Estresse ocupacional na administração pública Brasileira: quais os fatores impactantes? Revista de Administração Pública, Rio de Janeiro, v. 45, n. 3, p.751-774, jun. 2011. DOI: http://dx.doi.org/10.1590/s003476122011000300009.

BOZEMAN, B.; SCOTT, P. Bureaucratic Red Tape and Formalization: Untangling Conceptual Knots. The American Review of Public Administration, [s.I.], v. 26, n. 1, p.1-17, mar. 1996. DOI: http://dx.doi.org/10.1177/027507409602600101.

BREWER, G. A.; WALKER, R. M. Explaining variation in perceptions of red tape: a professionalism-marketization model. Public Administration, [s.I.], v. 88, n. 2, p.418-438, 14 mar. 2010. DOI: http://dx.doi.org/10.1111/j.1467-9299.2010.01827.x.

COOPER, C. L.; MARSHALL, J. Occupational sources of stress: a review of the literature relating to coronary heart disease and mental ill health. Journal of Occupational Psychology, [s.l.], v. 49, n. 1, p.11-28, mar. 1976. DOI: http://dx.doi.org/10.1111/j.20448325.1976.tb00325.x.

CROZIER, M. O fenômeno burocrático. Brasília, DF: Universidade de Brasília, p. 272, 1981.

DEHART-DAVIS, L. Red tape and public employees: does perceived rule dysfunction alienate managers? Journal of Public Administration Research and Theory, [s.I.], v. 15, n. 1, p.133148, 1 jan. 2005. Oxford University Press (OUP). DOI: http://dx.doi.org/10.1093/jopart/mui007.

HAIR, J. F. Análise multivariada de dados. 5. ed. Porto Alegre: Bookman, 2005.

HAMANN, D. J.; FOSTER, N. T. An exploration of job demands, job control, stress, and attitudes in public, nonprofit, and for-profit employees. Review of Public Personnel Administration, [s.I.], v. 34, n. 4, p.332-355, 25 jun. 2013. DOI: http://dx.doi.org/10.1177/0734371x13491119.

KARASEK, R. A. Job demands, job decision latitude, and mental strain: implications for job redesign. Administrative Science Quarterly, [s.I.], v. 24, n. 2, p.285-308, jun. 1979. DOI: JSTOR. http://dx.doi.org/10.2307/2392498.

KAUFMANN, W.; TUMMERS, L. The negative effect of red tape on procedural satisfaction. Public Management Review, [s.l.], v. 19, n. 9, p.1311-1327, 27 jul. 2016. DOI: http://dx.doi.org/10.1080/14719037.2016.1210907.

$\mathrm{KIM}, \mathrm{S}$. Factors affecting state government information technology employee turnover intentions. The American Review of Public Administration, [s.I.], v. 35, n. 2, p.137-156, jun. 2005. DOI: http://dx.doi.org/10.1177/0275074004273150. 
KIM, S. Y.; FERNANDEZ, S. Employee empowerment and turnover intention in the U.S. Federal Bureaucracy. The American Review of Public Administration, [s.I.], v. 47, n. 1, p.422, 28 jul. 2016. DOI: http://dx.doi.org/10.1177/0275074015583712.

LAMBERT, E. G.; HOGAN, N. L.; CAMP, S. D.; VENTURA, L. A. The impact of work-family conflict on correctional staff. Criminology \& Criminal Justice, [s.I.], v. 6, n. 4, p.371-387, nov. 2006. DOI: http://dx.doi.org/10.1177/1748895806068572.

LYRIO, M. V. L.; DELLAGNELO, E. H. L.; LUNKES, R. J. Proposta de um modelo de análise da flexibilização da burocracia em organizações públicas com base nas dimensões sugeridas por Volberda: o caso da secretaria de estado da administração de Santa Catarina. Administração Pública e Gestão Social, Viçosa, v. 9, n. 4, p.255-264, 1 out. 2017. DOI:

http://dx.doi.org/10.21118/apgs.v1i4.1048.

MAFFIA, L. N.; PEREIRA, L. Z. Estresse no trabalho: estudo com gestores públicos do Estado de Minas Gerais. Read. Revista Eletrônica de Administração, Porto Alegre, v. 20, n. 3, p.658680, dez. 2014. DOI: http://dx.doi.org/10.1590/1413-2311.0052014.47163.

MAKHIJA, P.; NAIDU, G. Occupational stress amongst women employees - a review of literature. Asia Pacific Journal of Research, v. 1, n. 43, August, p. 249-257, 2016.

MOON, M. J.; BRETSCHNEIBER, S. Does the perception of red tape constrain it innovativeness in organizations? unexpected results from a simultaneous equation model and implications. Journal of Public Administration Research and Theory, [s.I.], v. 12, n. 2, p.273-292, 1 abr. 2002. DOI: http://dx.doi.org/10.1093/oxfordjournals.jpart.a003532.

MOYNIHAN, D. P.; PANDEY, S. K. The role of organizations in fostering public service motivation. Public Administration Review, [s.I.], v. 67, n. 1, p.40-53, jan. 2007. DOI: http://dx.doi.org/10.1111/j.1540-6210.2006.00695.x.

OLIVIERI, C. Os controles políticos sobre a burocracia. Revista de Administração Pública, Rio de Janeiro, v. 45, n. 5, p.1395-1424, out. 2011. DOI: http://dx.doi.org/10.1590/s003476122011000500007.

PAIVA, K. C. M.; COUTO, J. H. Qualidade de vida e estresse gerencial "pós-choque de gestão": o caso da Copasa-MG. Revista de Administração Pública, Rio de Janeiro, v. 42, n. 6, p.11891211, dez. 2008. DOI: http://dx.doi.org/10.1590/s0034-76122008000600008.

PANDEY, S. K.; KINGSLEY, G. A. Examining red tape in public and private organizations: alternative explanations from a social psychological model. Journal of Public Administration Research and Theory, [s.I.], v. 10, n. 4, p.779-800, 1 out. 2000. DOI: http://dx.doi.org/10.1093/oxfordjournals.jpart.a024291.

PANDEY, S. K. MOYNIHAN, D. P. Bureaucratic red tape and organizational performance: testing the moderating role of culture and political support. In BOYNE, George A.; MEIER, Kenneth. J.; O'TOOLE JR., Laurence. J.; WALKER, Richard. M. Public service performance: perspectives on measurement and management. Cambridge, England, Cambridge University Press, p. 130-151, 2006. 
PANDEY, S. K.; SCOTT, P. G. Red Tape: A review and assessment of concepts and measures. Journal of Public Administration Research and Theory, [s.I.], v. 12, n. 4, p.553-580, 1 out. 2002. DOI: http://dx.doi.org/10.1093/oxfordjournals.jpart.a003547.

PARKER, D. F.; DECOTIIS, T. A. Organizational determinants of job stress. Organizational Behavior and Human Performance, [s.I.], v. 32, n. 2, p.160-177, out. 1983. DOI: http://dx.doi.org/10.1016/0030-5073(83)90145-9.

PIRES, R. R. C. Burocracia, discricionariedade e democracia: alternativas para o dilema entre controle do poder administrativo e capacidade de implementação. Cadernos Gestão Pública e Cidadania, São Paulo, v. 14, n. 54, p.147-187, 1 jan. 2009. DOI: http://dx.doi.org/10.12660/cgpc.v14n54.44190.

PIZZA JR., W. Burocracias e (des)burocratização. Revista de Administração Pública. Rio de Janeiro, v. 18, n. 3, p. 4-17, jul./set., 1984. Disponível em: http://bibliotecadigital.fgv.br/ojs/index.php/rap/article/view/10508. Acesso em: 20 dez. 2017.

SANTOS, E. A.; OLIVEIRA, R. M.; KONOPKA, R. Justiça organizacional e clima ético: percepção dos efeitos no estresse relacionado com o trabalho. Revista Capital Científico - Eletrônica, Guarapuava, v. 14, n. 3, p. 69-83, 2016. DOI: http://dx.doi.org/10.5935/21774153.20160021.

SANTOS, L. A. S.; MARQUES, D. B. Burocracia e economia dos custos de transação: uma análise da relação entre a Universidade Federal de Pernambuco e a Refinaria Abreu e Lima. Administração Pública e Gestão Social, Viçosa, v. 7, n. 1, jan./mar., p. 26-36, 2015. DOI: https://doi.org/10.21118/apgs.v7i1.509.

SARAIVA, L. A. S.; CAPELÃO, L. G. F. A nova administração pública e o foco no cidadão: burocracia X marketing? Revista de Administração Pública, Rio de Janeiro, v. 34, n. 2, 2000.

SARAIVA, L. A. S. Cultura organizacional em ambiente burocrático. Revista de Administração Contemporânea, Curitiba, v. 6, n. 1, p.187-207, abr. 2002 DOI: http://dx.doi.org/10.1590/s1415-65552002000100011.

SERT, A.; ELÇI, M.; USLU, T.; SENER, I. The effects of organizational justice and ethical climate on perceived work related stress. Procedia - Social and Behavioral Sciences, [s.I.], v. 150, p.1187-1198, set. 2014. DOI: http://dx.doi.org/10.1016/j.sbspro.2014.09.134.

SMITH, M. P. Alienation and bureaucracy: the role of participatory administration. Public Administration Review, [s.I.], v. 31, n. 6, p.658-664, nov. 1971. DOI: http://dx.doi.org/10.2307/974259.

TAMAYO, A. Prioridades axiológicas, atividade física e estresse ocupacional. Revista de Administração Contemporânea, Curitiba, v. 5, n. 3, p.127-147, dez. 2001 DOI: http://dx.doi.org/10.1590/s1415-65552001000300007. 
TENÓRIO, F. G. Weber e a burocracia. Revista do Serviço Público. Brasília, v. 48, n. 4, p. 7990, 1981. DOI: https://doi.org/10.21874/rsp.v38i4.2328.

WARBURTON, N. Uma breve história da filosofia. Porto Alegre, RS: L\&PM, p. 147-152, 2016.

WEBER, M. Economia e sociedade: fundamentos de sociologia compreensiva. Brasília, DF: Editora UnB, v. 2, p. 187-212, 1999.

WRIGHT, B. E. The role of work context in work motivation: a public sector application of goal and social cognitive theories. Journal of Public Administration Research and Theory, [s.l.], v. 14, n. 1, p.59-78, jan. 2004. DOI: http://dx.doi.org/10.1093/jopart/muh004.

ZEFFANE, R. Uncertainty, participation and alienation: lessons for workplace restructuring. International Journal of Sociology and Social Policy, [s.I.], v. 13, n. 5/6, p.22-52, maio 1993. DOI: http://dx.doi.org/10.1108/eb013174. 